\title{
Phase II study of trastuzumab with modified docetaxel, cisplatin, and 5 fluorouracil in metastatic HER2-positive gastric cancer
}

\author{
Sebastian Mondaca ${ }^{1}$ Matthew Margolis ${ }^{1} \cdot$ Francisco Sanchez-Vega $^{2,3} \cdot$ Philip Jonsson $^{2,4}$. Jamie C. Riches ${ }^{1}$. \\ Geoffrey Y. Ku ${ }^{1}$. Jaclyn F. Hechtman ${ }^{3}$. Yaelle Tuvy ${ }^{1} \cdot$ Michael F. Berger $^{2,3,5}$ - Manish A. Shah ${ }^{1}$. David P. Kelsen ${ }^{1}$. \\ David H. Ilson ${ }^{1} \cdot$ Kenneth $\mathrm{Yu}^{1}$ • Zoe Goldberg ${ }^{1}$ - Andrew S. Epstein ${ }^{1}$. Avni Desai ${ }^{1}$ - Vincent Chung ${ }^{6}$. Joanne F. Chou ${ }^{4}$. \\ Marinela Capanu ${ }^{4} \cdot$ David B. Solit ${ }^{1,2,3} \cdot$ Nikolaus Schultz $^{2,3} \cdot$ Yelena Y. Janjigian ${ }^{1}$
}

Received: 14 June 2018 / Accepted: 30 July 2018 / Published online: 7 August 2018

(c) The International Gastric Cancer Association and The Japanese Gastric Cancer Association 2018

\begin{abstract}
Background Trastuzumab with cisplatin and fluoropyrimidine is the standard treatment in metastatic HER2-positive gastric or gastroesophageal (GE) junction adenocarcinoma; however, there is limited data on the efficacy of trastuzumab in combination with a three-drug regimen in this setting. We examined the efficacy and safety of modified docetaxel, cisplatin and 5 fluorouracil (mDCF) plus trastuzumab in a single-arm multicenter phase II trial.

Methods Previously untreated patients with HER2-positive metastatic gastric or GE junction adenocarcinoma were treated with $\mathrm{mDCF}$ and trastuzumab every 2 weeks. The primary endpoint was 6-month progression-free survival (PFS); secondary endpoints included objective response rate, overall survival (OS), and toxicity.

Results We enrolled 26 patients with metastatic HER2-positive gastric or GE junction adenocarcinoma between February 2011 and June 2015. The median age of patients was 62 years; 96\% had a Karnofsky performance status equal to or greater than $80 \%$. With a median follow-up of 25.4 months, the 6-month PFS was $73 \%$ (95\% CI 51-86\%). The objective response rate was 65\%, the median PFS was 13 months (95\% CI 6.4-20.7) and the median OS was 24.9 months (95\% CI 14.4-42.5). Grade 3/4 toxicities included neutropenia (42\%), fatigue (23\%), and hypophosphatemia (15\%). There were no episodes of febrile neutropenia.

Conclusion The combination of mDCF and trastuzumab is effective and safe in patients with metastatic HER2-positive gastric or GE junction adenocarcinoma and can be considered as an option for selected patients. This trial is registered at ClinicalTrials.gov, number NCT00515411.
\end{abstract}

Keywords Gastric cancer · HER2 · Trastuzumab · Docetaxel

\section{Introduction}

Patients with metastatic gastric cancer (mGC) and gastroesophageal (GE) junction adenocarcinoma are treated with palliative chemotherapy consisting of platinum and fluoropyrimidine, which improves survival and quality of life [1]. Adding a third drug, most commonly a taxane, has shown

Electronic supplementary material The online version of this article (https://doi.org/10.1007/s10120-018-0861-7) contains supplementary material, which is available to authorized users.

Yelena Y. Janjigian

janjigiy@mskcc.org

Extended author information available on the last page of the article greater efficacy but is also associated with increased toxicity [2].

A significant fraction (20-25\%) of mGCs overexpress the receptor tyrosine kinase HER 2 often as a result of amplification of its gene, $E R B B 2$, and this receptor has been validated as a molecular target in the metastatic setting [3]. HER2 amplification is particularly frequent in the chromosomal instability subtype of gastric cancer and frequently co-occurs with TP53 mutations [4]. The HER2-blocking antibody trastuzumab has thus been combined with cisplatin and fluoropyrimidine to treat HER2-positive advanced gastric or GE junction adenocarcinoma; the ToGA trial showed that improvement in overall survival is particularly pronounced in patients with HER2 immunohistochemistry (IHC) $3+$ or HER2 IHC $2+$ and FISH-positive tumors [5, 6]. Since that 
trial, trastuzumab has been shown effective and safe in combination with other 2-drug chemotherapy regimens such as XELOX, FOLFOX, and S1 plus cisplatin as first-line therapy [7-12].

The combination of trastuzumab and docetaxel or paclitaxel has demonstrated synergy in HER2-overexpressing human gastric cancer xenograft models [13]. Recently we reported that in patients with $\mathrm{mGC}$ modified docetaxel, cisplatin, and 5 fluorouracil (mDCF) was tolerable with similar efficacy as parent DCF [14]. When trastuzumab was approved for HER2 amplified metastatic gastric cancer, the mDCF protocol was amended to allow HER2-positive patients to receive this biologic with $\mathrm{mDCF}$, as a separate and independent cohort. Therefore, the aim of this study is to describe the efficacy of mDCF with trastuzumab as measured by 6-month PFS as well as its safety profile in HER2-positive mGC patients.

\section{Methods}

\section{Patients}

This is a single-arm multicenter phase II trial for patients with metastatic gastric or gastroesophageal (GE) junction adenocarcinoma found to be HER2-positive, defined as IHC staining intensity rated as $3+$ on a scale of 0 to $3+$ or IHC $2+$ with ERBB2 gene amplification confirmed by FISH. Inclusion criteria were radiographically evaluable disease, Karnofsky performance status $\geq 70 \%$, and adequate organ function defined as: hemoglobin $\geq 9.0 \mathrm{~g} / \mathrm{dL}$, absolute neutrophil count $\geq 1500$ cells/L, platelet count $\geq 100,000 / \mathrm{L}$, serum creatinine $\leq 1.5 \mathrm{mg} / \mathrm{dL}$, total bilirubin $\leq 1.5 \mathrm{mg} / \mathrm{dL}$, AST, ALT, and alkaline phosphatase within eligible ranges (Online Resource 1) and baseline left ventricular ejection fraction $\geq 50 \%$. In patients with serum creatinine of $1.2-1.5 \mathrm{mg} / \mathrm{dL}$, creatinine clearance (calculated or observed) must have been $\geq 50 \mathrm{~mL} / \mathrm{min}$. Anticoagulation was allowed. Patients were excluded if they had received prior chemotherapy for metastatic or unresectable disease, received prior docetaxel or cisplatin in the perioperative or adjuvant setting. Other exclusion criteria were: brain or central nervous system metastasis, significant cardiovascular disease (unstable angina, myocardial infarction, stroke, or cerebrovascular accident within 6 months of study entry), or clinically significant hearing loss or tinnitus. Cancer diagnose within the past 3 years (excluding basal cell carcinoma of skin, cervical carcinoma in situ, or non-metastatic prostate cancer) was also an exclusion criterion. All patients signed written informed consent prior to study enrollment. This trial is registered at ClinicalTrials.gov (NCT00515411).

This study protocol was approved by the institutional review boards at Memorial Sloan Kettering Cancer Center
(MSKCC) and City of Hope Cancer Center. The study was monitored quarterly by the MSKCC Data and Safety Monitoring Board and MSKCC Therapeutic Response Review Committee. This study was done in accordance with the principles of the Declaration of Helsinki.

\section{Treatment plan and tumor assessment}

Patients were treated with $\mathrm{mDCF}$ and trastuzumab and the specific modifications to parent DCF were: a de Gramont schedule for 5 fluorouracil (5-FU) administration; reduced bi-monthly doses of cisplatin and docetaxel; the administration of docetaxel and cisplatin on consecutive days instead of immediately following one another. The regimen consists in docetaxel $40 \mathrm{mg} / \mathrm{m}^{2}$ day 1 , cisplatin $40 \mathrm{mg} / \mathrm{m}^{2}$ day 2 ,

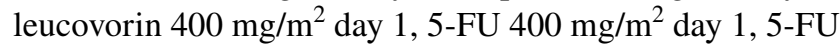
$1000 \mathrm{mg} / \mathrm{m}^{2} /$ day (continuous intravenous infusion) days 1 and 2 , on a 2 -week cycle. Trastuzumab was also administered on day 1 of the 2 -week cycle, with an initial loading dose of $6 \mathrm{mg} / \mathrm{kg}$ in cycle 1 and $4 \mathrm{mg} / \mathrm{kg}$ thereafter. Cisplatin was recommended for up to 12 cycles and the rest of the regimen until disease progression, unacceptable toxicity or withdrawal of consent. Tumor growth was followed radiographically by CT of the chest, abdomen, and pelvis, performed every 6 weeks initially and then every 12 weeks after 9 months on therapy. Response and progression were evaluated using Response Evaluation Criteria in Solid Tumors (RECIST) criteria [15].

\section{Toxicity evaluation and dose attenuation}

All adverse events were graded according to the National Cancer Institute Common Toxicity Criteria for Adverse Events (v 3.0). Doses were reduced following a standard scale in case of adverse events. Left ventricular function was assessed by echocardiogram every 12 weeks while on study, and trastuzumab was stopped if a patient developed symptomatic congestive heart failure (CHF), or any asymptomatic decrease in left ventricular ejection fraction (LVEF) below $50 \%$ with an absolute reduction of at least $10 \%$ from baseline.

\section{Statistics}

The primary endpoint of this study was 6-month progression-free survival (PFS). PFS was measured from the start of treatment to the date of either documented disease progression or death, whichever occurred first. Given that the 6-month PFS of the parent DCF regimen is approximately $43 \%$ [2], we estimated using exact single-stage binomial design that a sample size of 35 HER2-positive patients would be sufficient to differentiate between 6-month PFS of 43 and $64 \%$ with type I and II error rates of $10 \%$ each 
[16]. The modified DCF plus trastuzumab regimen would be considered promising if 19 out of 35 patients were alive and progression free at 6 months. We employed an early stopping rule for safety as previously described [14]. We defined an acceptable grade 3-4 adverse event rate of 50\% and an unacceptable grade 3-4 adverse event rate of $70 \%$ in the first 3 months of therapy. Other secondary endpoints included response rate, median PFS, and overall and 1- and 2-year survival. The median PFS and median overall survival (OS) were estimated using the Kaplan-Meier method, and 95\% confidence intervals were based on the sign test [17]. All statistical analyses were performed using SAS version 9.3 (SAS Institute, INC., Cary, NC, USA). All $P$ values were two sided. $P$ values of $<0.05$ were considered to indicate statistical significance.

\section{Sequencing and bioinformatics}

Participants enrolled after February 2014 were eligible to consent to prospective tumor genomic profiling using the next generation sequencing (NGS) platform MSK-IMPACT. This assay was conducted as previously described [18, 19] and was performed in a CLIA-certified laboratory, using a 341-gene panel. MSK-IMPACT detects mutations, small insertions and deletions, copy number alterations and selected structural rearrangements. We also assessed tumors for microsatellite instability (MSI) using the MSIsensor method, and samples with a score $\geq 10$ were classified as MSI-high [20]. All samples were collected for analysis before starting anti-HER2 treatment. The log-rank test was used to test for associations between specific genomic alterations and PFS.

\section{Results}

\section{Patients}

Twenty-six patients with HER2-positive metastatic gastric or GE junction adenocarcinoma were enrolled between February 2011 and June 2015. Two patients were enrolled at a collaborative site and the rest were treated at MSKCC. The study was closed before reaching estimated sample size because of slow accrual. The median age at the time of enrollment was 62 years (range 35-77 years) and 25 patients (96\%) had a Karnofsky performance status equal to or greater than $80 \%$ (Table 1). In this cohort 18 patients' tumors (69\%) qualified as HER2-positive based on IHC $3+$ and 8 (31\%) based on IHC $2+$ in combination with FISH (+). A focal staining pattern was described in 2 patients (13\%) with IHC scores of $3+$ and in $3(38 \%)$ with $2+$.
Table 1 Baseline patient characteristics $(n=26)$

\begin{tabular}{lc}
\hline & No. $(\%)$ \\
\hline Age, years ${ }^{\mathrm{a}}$ & $62(35-77)$ \\
Sex & \\
Male & $23(88.5)$ \\
Female & $3(11.5)$ \\
Stage at diagnosis & \\
Stage I/II/III & $3(11.5)$ \\
Stage IV & $23(88.5)$ \\
Tumor location & \\
GE junction & $15(58)$ \\
Stomach & $11(42)$ \\
Baseline KPS (\%) & \\
$\geq 80 \%$ & $25(96)$ \\
$70 \%$ & $1(4)$ \\
Lauren classification & \\
Intestinal & $13(50)$ \\
Mixed & $4(15)$ \\
Diffuse & $7(27)$ \\
Unknown & $2(8)$ \\
HER2 status & \\
IHC $3+$ & $18(69)$ \\
IHC 2 +/FISH (+) & $8(31)$ \\
\hline$K P S$ Karnsky perfor &
\end{tabular}

KPS Karnofsky performance status

${ }^{a}$ Median (range)

Table 2 Response rate according to RECIST criteria

\begin{tabular}{lcl}
\hline Best response & $n(\%)$ & $\begin{array}{l}\text { Duration } \\
\text { (months) }\end{array}$ \\
\hline Complete response & $1(4)$ & 77 \\
Partial response & $16(62)$ & 19 \\
Stable disease & $6(23)$ & 6 \\
Disease progression $^{\text {No further evaluation }}{ }^{\mathrm{a}}$ & $1(4)$ & $(-)$ \\
\hline
\end{tabular}

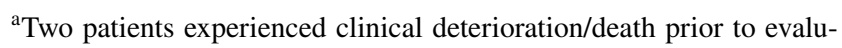
ation by imaging

\section{Treatment and outcomes}

All 26 patients received at least 1 cycle of $\mathrm{mDCF}$ and trastuzumab; the median number of chemotherapy cycles was 21 (range 1-153). The mean relative dose intensities for docetaxel, cisplatin, bolus 5-FU, infusional 5-FU and trastuzumab were $69 \%, 50 \%, 90 \%, 93 \%$, and $100 \%$, respectively. In the intention-to-treat population, the objective response rate was $65 \%$ (95\% CI 46-80\%) (Table 2); response is quantified as best percentage change in tumor diameters in Fig. 1. Two patients experienced clinical deterioration or death prior to evaluation by imaging. The median follow-up 


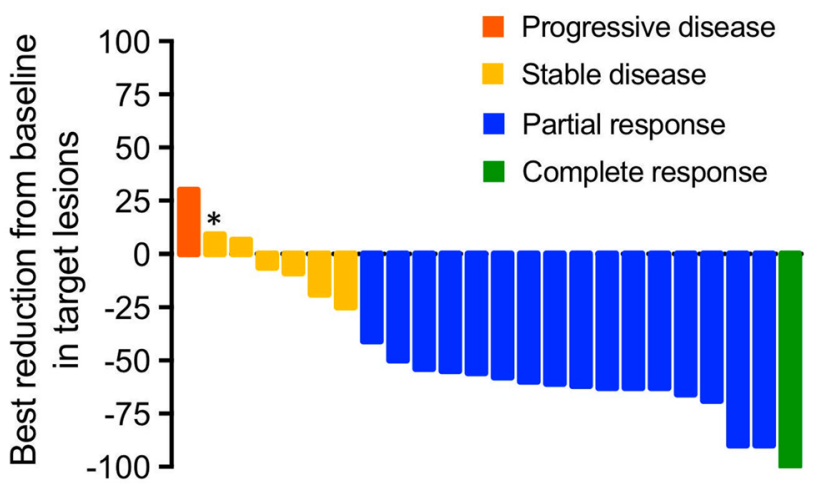

Fig. 1 Treatment response of each patient, quantified as best percentage change in sum of longest tumor diameters of target lesions. *One patient with a non-target 14 -mm lymph node (short axis) was included in this analysis

was 25.4 months; at 6 months, 19 of 26 patients were alive and progression-free, which represents a 6-month PFS of $73 \%$ (95\% CI 51-86). The median PFS was 13 months (95\% CI 6.4-20.7) and the median OS was 24.9 months (95\% CI 14.4-42.5) (Fig. 2). The 1- and 2-year survival was 73 and $56 \%$, respectively. Patients received second, third and fourth lines of systemic treatment in $81 \%, 58 \%$, and $12 \%$ of cases, respectively. Four patients $(15 \%)$ were later treated with immune checkpoint inhibitors and $14(54 \%)$ received trastuzumab after progression. Subsequent systemic therapies are listed in Table 3.

\section{Genomic analysis}

The tumors of 11 patients were prospectively genomically profiled at a mean sequencing coverage of $828 \times$. Figure 3 provides a summary of mutations detected by targeted NGS. Sequenced samples were from primary tumors in 8 patients and metastases in 3 . All samples were classified
Table 3 Subsequent lines of therapy

\begin{tabular}{|c|c|}
\hline & $n(\%)$ \\
\hline \multicolumn{2}{|l|}{ Second-line therapy } \\
\hline Fluoropyrimidines & $4(15)$ \\
\hline Irinotecan & $11(42)$ \\
\hline Platin $^{\mathrm{a}}$ & $10(38)$ \\
\hline Taxane & $3(11.5)$ \\
\hline Paclitaxel-ramucirumab & 0 \\
\hline Checkpoint inhibitors & $3(11.5)$ \\
\hline Trastuzumab & $12(46)$ \\
\hline Other & $3(11.5)$ \\
\hline \multicolumn{2}{|l|}{ Third-line therapy } \\
\hline Fluoropyrimidines & $6(23)$ \\
\hline Irinotecan & $4(15)$ \\
\hline Platin $^{\mathrm{a}}$ & $9(35)$ \\
\hline Taxane & $2(8)$ \\
\hline Paclitaxel-ramucirumab & $1(4)$ \\
\hline Checkpoint inhibitors & $2(8)$ \\
\hline Trastuzumab & $8(31)$ \\
\hline Other & $1(4)$ \\
\hline \multicolumn{2}{|l|}{ Fourth-line therapy } \\
\hline Fluoropyrimidines & $1(4)$ \\
\hline Irinotecan & 0 \\
\hline Platin $^{\mathrm{a}}$ & $1(4)$ \\
\hline Taxane & 0 \\
\hline Paclitaxel-ramucirumab & $1(4)$ \\
\hline Checkpoint inhibitors & 0 \\
\hline Trastuzumab & $2(8)$ \\
\hline Other & $1(4)$ \\
\hline
\end{tabular}

${ }^{a}$ Includes cisplatin, carboplatin, and oxaliplatin

as microsatellite stable (MSI score $<10$ ). The most frequently altered genes were TP53, in 11 patients $(100 \%)$, and $C D K N 2 A$, in 3 patients (27\%). NGS confirmed
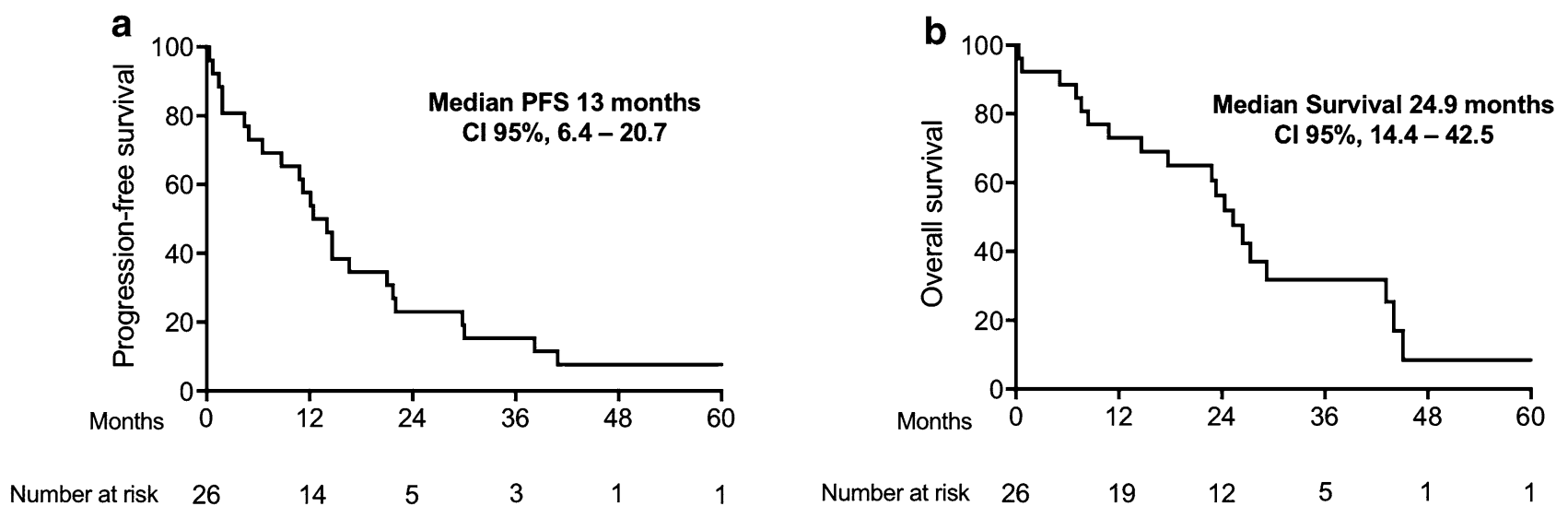

Fig. 2 Outcomes in patients treated with first-line mDCF and trastuzumab. a Progression-free survival (PFS) and b overall survival (OS) 


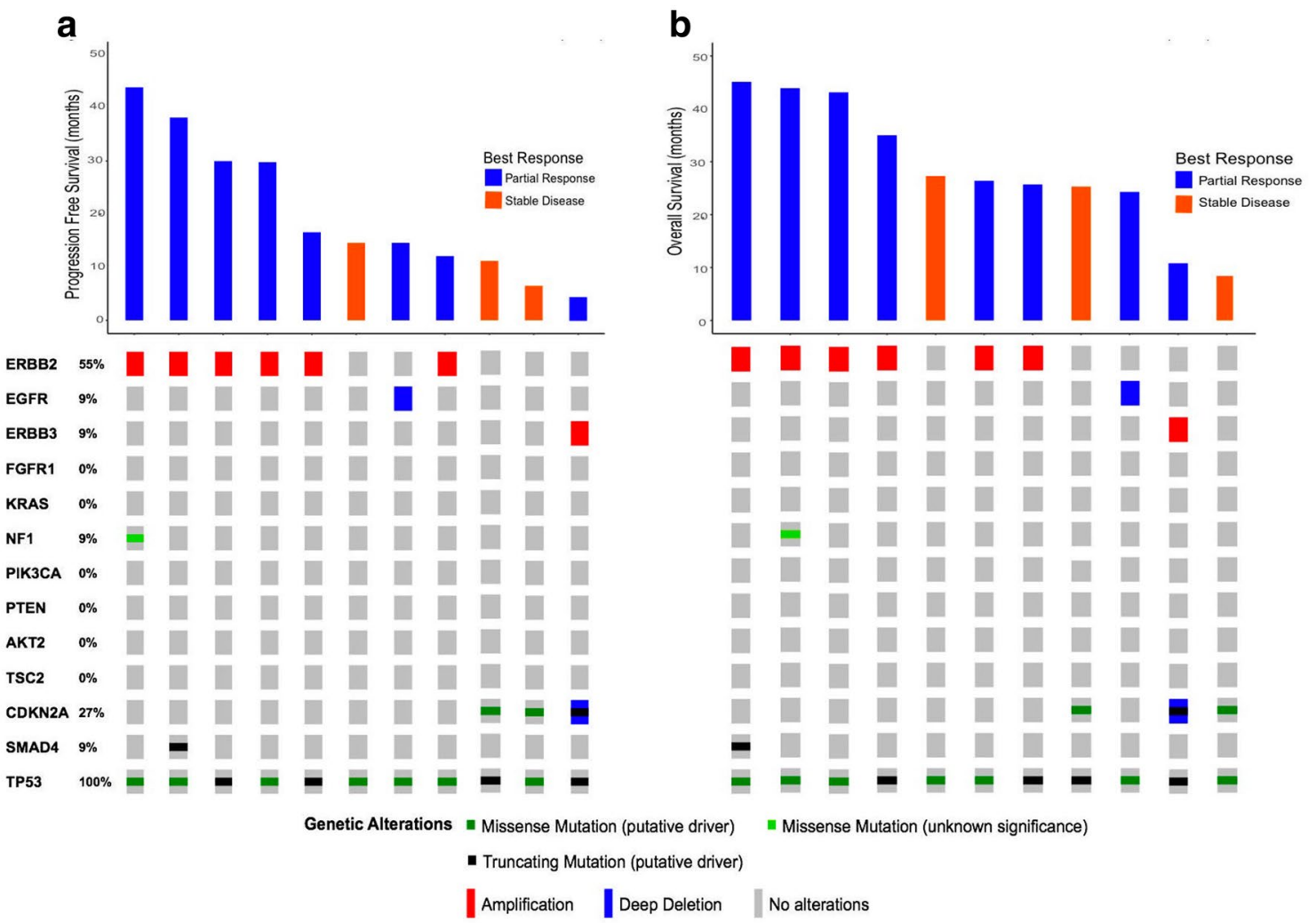

Fig. 3 Genomic analysis of advanced HER2-positive gastric cancer. Oncoprint depicts alterations in selected genes of interest in 11 HER2-positive gastric cancer patients treated with $\mathrm{mDCF}$ and trastu-

$E R B B 2$ amplification in 6 patients $(55 \%)$ and the remaining 5 patients had significantly shorter PFS compared to patients with ERBB2 amplified tumors (median PFS, 11 vs. 30 months, $p=0.0072$ ). Among these 5 patients, HER2 staining was only focally positive in 1 , and FISH amplification was borderline positive in 2 (Online Resource 2).

\section{Toxicity}

Grade 3/4 non-hematologic toxicities included fatigue (23\%), hypersensitivity reaction $(11.5 \%)$, dehydration $(11.5 \%)$, diarrhea $(8 \%)$, and gastrointestinal hemorrhage (8\%). Three patients (11.5\%) came off study because of toxicity, two because of grade 3 gastrointestinal bleeding and one because of persistent grade 2 anorexia in the setting of controlled disease. Grade 3/4 neutropenia occurred in 11 patients (42\%). One patient (4\%) had asymptomatic drop in LVEF below $50 \%$ and none had symptomatic CHF. There were no episodes of febrile neutropenia or drug-related mortality. Attributable adverse events are listed in Table 4. zumab. Correlation with progression-free survival (a) and overall survival (b) are described

\section{Discussion}

After the seminal ToGA trial, the combination of two chemotherapy drugs and trastuzumab became the standard of care in HER2-amplified mGC. In this study we found that in selected patients, the combination of taxane, fluoropyrimidine, and cisplatin with trastuzumab is also feasible. Furthermore, we observed a promising activity of this regimen with a 6-month PFS of $73 \%$. Notably, grade $3 / 4$ toxicity was low and there were no cases of febrile neutropenia. This trial is a continuation of a randomized phase II trial of $\mathrm{mDCF}$ vs. original DCF in unselected mGC [14]. Our findings further support the efficacy of this regimen and reveal no new safety concerns. Other small series also have shown encouraging results with the combination of trastuzumab with a threedrug regimen, such as docetaxel, cisplatin and S1 [21], and docetaxel, oxaliplatin, capecitabine, bevacizumab [22]. In our cohort $96 \%$ of patients had KPS $\geq 80 \%$ which may have allowed patients to tolerate treatment for a longer period of time (median number of cycles 21 ) leading to a better than expected PFS. Remarkably, 81 and $58 \%$ of patients received second- and third-line systemic therapy, respectively, suggesting that in appropriately selected patients this regimen can maximize exposure to active treatments. 
Table 4 Toxicity possibly, probably, or definitely related to $\mathrm{mDCF}$ and trastuzumab $(n=26)$

\begin{tabular}{lcl}
\hline & Any grade $n(\%)$ & Grade $3-4 n(\%)$ \\
\hline Non-hematologic & & \\
Fatigue & $13(50)$ & $6(23)$ \\
Hypophosphatemia & $4(15)$ & $4(15)$ \\
Hypokalemia & $5(19)$ & $3(11.5)$ \\
Hyponatremia & $6(23)$ & $1(4)$ \\
Hyperglycemia & $7(27)$ & $1(4)$ \\
Hypersensitivity reaction & $8(31)$ & $3(11.5)$ \\
Dehydration & $5(19)$ & $3(11.5)$ \\
Diarrhea & $10(38)$ & $2(8)$ \\
GI hemorrhage & $2(8)$ & $2(8)$ \\
Anorexia & $3(11.5)$ & $1(4)$ \\
Hyperbilirubinemia & $3(11.5)$ & $1(4)$ \\
Hypoalbuminemia & $2(8)$ & $1(4)$ \\
Epiphora & $7(27)$ & $1(4)$ \\
Nausea & $11(42)$ & $1(4)$ \\
Syncope & $1(4)$ & $1(4)$ \\
Thromboembolism & $2(8)$ & $1(4)$ \\
Hematologic & $16(62)$ & $11(42)$ \\
Neutropenia & $8(31)$ & $8(31)$ \\
Lymphopenia & $17(65)$ & $7(27)$ \\
Anemia & $13(50)$ & $1(4)$ \\
Thrombocytopenia & & \\
\hline
\end{tabular}

GI gastrointestinal

Our exploratory sequencing analysis failed to confirm $E R B B 2$ amplification in 5 of 11 patients and that group derived limited benefit from trastuzumab-based therapy. This discordance could be attributed to intra-tumor heterogeneity for $E R B B 2$ amplification [23], which could explain the lack of benefit in clinical trials of other anti-HER2 therapies such as higher doses of trastuzumab [24]. As expected, in our cohort all patients had a signature of chromosomal instability with high frequency of TP53 mutations. A limitation of this study is that enrollment terminated prior completing accrual, however, the study reached its primary endpoint as 19 patients were progression-free at 6 months (73\%). HER2-overexpressing mGC invariably progresses and develops resistance to trastuzumab. Based on preclinical studies showing that HER2 inhibition can promote $\mathrm{T}$ cell activation [25], ongoing studies are investigating the combination of anti-HER2 treatment and immunotherapy in patients with HER2-positive mGC. These include two phase II trials exploring the combination of pembrolizumab with trastuzumab and chemotherapy in HER2-positive mGC (NCT02954536, NCT02901301). In conclusion, this phase II trial demonstrated the efficacy and safety of the combination of $\mathrm{mDCF}$ and trastuzumab in HER2-positive mGC and adds further evidence for this three-drug regimen as a standard option for fit gastric cancer patients.

Funding This study was funded by a 2013 Conquer Cancer Foundation ASCO Career Development Award (Y.Y.J.) and by Genentech.

\section{Compliance with ethical standards}

Conflict of interest Y. Y. Janjigian has received research funding from Boehringer Ingelheim, Bayer, Genentech/Roche, Bristol-Myers Squibb, Eli Lilly, and Merck and serves on advisory boards for Merck Serono, Bristol-Myers Squibb, Eli Lilly, Pfizer, and Merck.

Ethical standards The present study protocol was approved by the institutional review boards at Memorial Sloan Kettering Cancer Center (MSKCC) and City of Hope Cancer Center. All patients signed written informed consent prior to study enrollment. The study was monitored quarterly by the MSKCC Data and Safety Monitoring Board and MSKCC Therapeutic Response Review Committee. This study was done in accordance with the principles of the Declaration of Helsinki.

\section{References}

1. Wagner AD, Syn NL, Moehler M, Grothe W, Yong WP, Tai BC, et al. Chemotherapy for advanced gastric cancer. Cochrane Database Syst Rev. 2017. https://doi.org/10.1002/14651858.CD004 064.pub4. (Epub 2017/08/29, PubMed PMID: 28850174).

2. Van Cutsem E, Moiseyenko VM, Tjulandin S, Majlis A, Constenla M, Boni C, et al. Phase III study of docetaxel and cisplatin plus fluorouracil compared with cisplatin and fluorouracil as first-line therapy for advanced gastric cancer: a report of the V325 Study Group. J Clin Oncol. 2006;24(31):4991-7. https://doi. org/10.1200/JCO.2006.06.8429. (PubMed PMID: 17075117).

3. Van Cutsem E, Bang YJ, Feng-Yi F, Xu JM, Lee KW, Jiao SC, et al. HER2 screening data from ToGA: targeting HER2 in gastric and gastroesophageal junction cancer. Gastric Cancer. 2015;18(3):476-84. https://doi.org/10.1007/s10120-014-0402y. (PubMed PMID: 25038874; PubMed Central PMCID: PMC4511072, Epub 2014/07/20).

4. Cancer Genome Atlas Research Network. Comprehensive molecular characterization of gastric adenocarcinoma. Nature. 2014;513(7517):202-9. https://doi.org/10.1038/nature1348 0. (PubMed PMID: 25079317; PubMed Central PMCID: PMC4170219).

5. Bang YJ, Van Cutsem E, Feyereislova A, Chung HC, Shen L, Sawaki A, et al. Trastuzumab in combination with chemotherapy versus chemotherapy alone for treatment of HER2positive advanced gastric or gastro-oesophageal junction cancer (ToGA): a phase 3, open-label, randomised controlled trial. Lancet. 2010;376(9742):687-97. https://doi.org/10.1016/ S0140-6736(10)61121-X. (PubMed PMID: 20728210, Epub 2010/08/19).

6. Satoh T, Bang YJ, Gotovkin EA, Hamamoto Y, Kang YK, Moiseyenko VM, et al. Quality of life in the trastuzumab for gastric cancer trial. Oncologist. 2014;19(7):712-9. https://doi.org/10.1634/ theoncologist.2014-0058. (PubMed PMID: 24951609; PubMed Central PMCID: PMC4077451).

7. Kataoka H, Mori Y, Shimura T, Nishie H, Natsume M, Mochizuki $\mathrm{H}$, et al. A phase II prospective study of the trastuzumab combined with 5-weekly S-1 and CDDP therapy for HER2positive advanced gastric cancer. Cancer Chemother Pharmacol. 
2016;77(5):957-62. https://doi.org/10.1007/s00280-016-3013-y. (PubMed PMID: 27002325, Epub 2016/03/22).

8. Ryu MH, Yoo C, Kim JG, Ryoo BY, Park YS, Park SR, et al. Multicenter phase II study of trastuzumab in combination with capecitabine and oxaliplatin for advanced gastric cancer. Eur J Cancer. 2015;51(4):482-8. https://doi.org/10.1016/j.ejca.2014.12.015. (PubMed PMID: 25661103, Epub 2015/02/03).

9. Gong J, Liu T, Fan Q, Bai L, Bi F, Qin S, et al. Optimal regimen of trastuzumab in combination with oxaliplatin/ capecitabine in first-line treatment of HER2-positive advanced gastric cancer (CGOG1001): a multicenter, phase II trial. BMC Cancer. 2016;16:68. https://doi.org/10.1186/s12885-016-20929. (PubMed PMID: 26857702; PubMed Central PMCID: PMC4746787, Epub 2016/02/08).

10. Soularue É, Cohen R, Tournigand C, Zaanan A, Louvet C, Bachet $\mathrm{JB}$, et al. Efficacy and safety of trastuzumab in combination with oxaliplatin and fluorouracil-based chemotherapy for patients with HER2-positive metastatic gastric and gastro-oesophageal junction adenocarcinoma patients: a retrospective study. Bull Cancer. 2015;102(4):324-31. https://doi.org/10.1016/j.bulca n.2014.08.001. (Epub 2015/03/03, PubMed PMID: 25744576).

11. Miura Y, Sukawa Y, Hironaka S, Mori M, Nishikawa K, Tokunaga S, et al. Five-weekly S-1 plus cisplatin therapy combined with trastuzumab therapy in HER2-positive gastric cancer: a phase II trial and biomarker study (WJOG7212G). Gastric Cancer. 2018;21(1):84-95. https://doi.org/10.1007/s10120-017-0725-6. (PubMed PMID: 28497176, Epub 2017/05/13).

12. Kurokawa Y, Sugimoto N, Miwa H, Tsuda M, Nishina S, Okuda $\mathrm{H}$, et al. Phase II study of trastuzumab in combination with S-1 plus cisplatin in HER2-positive gastric cancer (HERBIS-1). Br J Cancer. 2014;110(5):1163-8. https://doi.org/10.1038/bjc.2014.18. (PubMed PMID: 24473399; PubMed Central PMCID: PMC3950868, Epub 2014/01/30).

13. Fujimoto-Ouchi K, Sekiguchi F, Yasuno H, Moriya Y, Mori K, Tanaka Y. Antitumor activity of trastuzumab in combination with chemotherapy in human gastric cancer xenograft models. Cancer Chemother Pharmacol. 2007;59(6):795-805. https://doi. org/10.1007/s00280-006-0337-z. (PubMed PMID: 17031648, Epub 2006/10/10).

14. Shah MA, Janjigian YY, Stoller R, Shibata S, Kemeny M, Krishnamurthi $S$, et al. Randomized multicenter phase II study of modified docetaxel, cisplatin, and fluorouracil (DCF) versus DCF plus growth factor support in patients With metastatic gastric adenocarcinoma: a study of the US Gastric Cancer Consortium. J Clin Oncol. 2015. https://doi.org/10.1200/JCO.2015.60.7465. (PubMed PMID: 26438119).

15. Therasse P, Arbuck SG, Eisenhauer EA, Wanders J, Kaplan RS, Rubinstein L, et al. New guidelines to evaluate the response to treatment in solid tumors. European Organization for Research and Treatment of Cancer, National Cancer Institute of the United States, National Cancer Institute of Canada. J Natl Cancer Inst. 2000;92(3):205-16. (PubMed PMID: 10655437).

16. Chow S-C. Sample size calculations in clinical research. Boca Raton, CRC Press; 2003.

17. Anderson JR, Bernstein L, Pike MC. Approximate confidence intervals for probabilities of survival and quantiles in life-table analysis. Biometrics. 1982;38(2):407-16. (PubMed PMID: 7115871)

18. Cheng DT, Mitchell TN, Zehir A, Shah RH, Benayed R, Syed A, et al. Memorial Sloan Kettering-Integrated Mutation Profiling of Actionable Cancer Targets (MSK-IMPACT): a hybridization capture-based next-generation sequencing clinical assay for solid tumor molecular oncology. J Mol Diagn. 2015;17(3):251-64. https://doi.org/10.1016/j.jmoldx.2014.12.006. (PubMed PMID: 25801821, Epub 2015/03/20).

19. Zehir A, Benayed R, Shah RH, Syed A, Middha S, Kim HR, et al. Mutational landscape of metastatic cancer revealed from prospective clinical sequencing of 10,000 patients. Nat Med. 2017;23(6):703-13. https://doi.org/10.1038/nm.4333. (PubMed PMID: 28481359; PubMed Central PMCID: PMC5461196, Epub 2017/05/08).

20. Middha S, Zhang L, Nafa K, Jayakumaran G, Wong D, Kim HR, et al. Reliable pan-cancer microsatellite instability assessment by using targeted next-generation sequencing data. JCO Precis Oncol. 2017;1:1-17. https://doi.org/10.1200/po.17.00084.

21. Mitsui Y, Sato Y, Miyamoto H, Fujino Y, Takaoka T, Miyoshi J, et al. Trastuzumab in combination with docetaxel/cisplatin/S-1 (DCS) for patients with HER2-positive metastatic gastric cancer: feasibility and preliminary efficacy. Cancer Chemother Pharmacol. 2015;76(2):375-82. https://doi.org/10.1007/s00280-0152807-7. (PubMed PMID: 26099968, Epub 2015/06/23).

22. Meulendijks D, Beerepoot LV, Boot H, de Groot JW, Los M, Boers JE, et al. Trastuzumab and bevacizumab combined with docetaxel, oxaliplatin and capecitabine as first-line treatment of advanced HER2-positive gastric cancer: a multicenter phase II study. Investig New Drugs. 2016;34(1):119-28. https://doi.org/10.1007/s1063 7-015-0309-4. (PubMed PMID: 26643663, Epub 2015/12/08).

23. Janjigian YY, Sanchez-Vega F, Jonsson P, Chatila WK, Hechtman JF, Ku GY, et al. Genetic predictors of response to systemic therapy in esophagogastric cancer. Cancer Discov. 2018;8(1):4958. https://doi.org/10.1158/2159-8290.CD-17-0787. (PubMed PMID: 29122777; PubMed Central PMCID: PMC5813492, Epub 2017/11/11).

24. Shah MA, Xu RH, Bang YJ, Hoff PM, Liu T, Herráez-Baranda LA, et al. HELOISE: Phase IIIb randomized multicenter study comparing standard-of-care and higher-dose trastuzumab regimens combined with chemotherapy as first-line therapy in patients with human epidermal growth factor receptor 2-positive metastatic gastric or gastroesophageal junction adenocarcinoma. J Clin Oncol. 2017;35(22):2558-67. https://doi.org/10.1200/ JCO.2016.71.6852. (PubMed PMID: 28574779, Epub 2017/06/02).

25. Wolpoe ME, Lutz ER, Ercolini AM, Murata S, Ivie SE, Garrett ES, et al. HER-2/neu-specific monoclonal antibodies collaborate with HER-2/neu-targeted granulocyte macrophage colonystimulating factor secreting whole cell vaccination to augment $\mathrm{CD} 8+\mathrm{T}$ cell effector function and tumor-free survival in Her-2/ neu-transgenic mice. J Immunol. 2003;171(4):2161-9. (Epub 2003/08/07, PubMed PMID: 12902523). 


\section{Affiliations}

Sebastian Mondaca ${ }^{1} \cdot$ Matthew Margolis ${ }^{1} \cdot$ Francisco Sanchez-Vega $^{2,3} \cdot$ Philip Jonsson $^{2,4} \cdot$ Jamie C. Riches $^{1}$. Geoffrey Y. Ku ${ }^{1}$ - Jaclyn F. Hechtman ${ }^{3} \cdot$ Yaelle Tuvy $^{1} \cdot$ Michael F. Berger $^{2,3,5} \cdot$ Manish A. Shah $^{1} \cdot$ David P. Kelsen $^{1}$. David H. Ilson ${ }^{1} \cdot$ Kenneth Yu ${ }^{1}$. Zoe Goldberg ${ }^{1}$. Andrew S. Epstein ${ }^{1}$. Avni Desai ${ }^{1}$ - Vincent Chung ${ }^{6}$. Joanne F. Chou ${ }^{4}$. Marinela Capanu ${ }^{4}$. David B. Solit ${ }^{1,2,3}$ - Nikolaus Schultz ${ }^{2,3}$. Yelena Y. Janjigian ${ }^{1}$

1 Department of Medicine, Memorial Sloan Kettering Cancer Center and Weill Cornell Medical College, 300 E. 66th Street, Room 1033, New York, NY 10065, USA

2 Human Oncology and Pathogenesis Program, Memorial Sloan Kettering Cancer Center and Weill Cornell Medical College, New York, NY, USA

3 Marie-Josée and Henry R. Kravis Center for Molecular Oncology, Memorial Sloan Kettering Cancer Center and Weill Cornell Medical College, New York, NY, USA
4 Department of Epidemiology-Biostatistics, Memorial Sloan Kettering Cancer Center and Weill Cornell Medical College, New York, NY, USA

5 Physiology, Biophysics and Systems Biology Program, Weill Cornell Medical College, New York, NY, USA

6 Department of Medical Oncology and Therapeutics Research, City of Hope Cancer Center, Duarte, CA, USA 